\title{
The Product of Two Functions Using Positive Linear Operators
}

\author{
ADRIAN HOLHOŞ*
}

ABSTRACT. In this paper, we estimate the speed of convergence of the difference $L_{n}(f g)-\left(L_{n} f\right) \cdot\left(L_{n} g\right)$ towards 0 , where $\left(L_{n}\right)$ are positive linear operators used in the approximation of continuous functions. We also study in what conditions the formula $L_{n}^{\prime}(f g)-f L_{n}^{\prime} g-g L_{n}^{\prime} f \rightarrow 0$ holds true.

Keywords: Positive linear operators, exponential type operators, Voronovskaya formula, Chebyshev-Grüss functional, Baskakov operators, Jain operators, Balász operators.

2010 Mathematics Subject Classification: 41A36, 41A25.

\section{INTRODUCTION}

In the last period of time, it was investigated the following difference

$$
L_{n}(f g)(x)-\left(L_{n} f\right)(x) \cdot\left(L_{n} g\right)(x),
$$

a generalization to positive linear operators of an expression appearing in the classical inequalities of Chebyshev [15] and Grüss [24]. Starting with the papers [10, 19] and [7, 33], these celebrated inequalities were extended to the case of positive linear functionals and positive linear operators. Bounds for this difference were given using different methods (see [22, 20, 21]). Asymptotic results of Voronovskaya type for this Chebyshev-Grüss quantity were obtained in $[18,6,16,35,9,30]$ for different operators.

In this paper, we give a quantitative result of Voronovskaya type for the Chebyshev-Grüss expression for a large class of positive linear operators and for a large class of continuous functions. Our results, presented in Section 3, do not need as in $[9,30]$ the hypothesis of the existence of the second derivatives of the functions involved.

In Section 4, we study in what conditions do the differentiation formula $L_{n}^{\prime}(f g)-f L_{n}^{\prime} g-$ $g L_{n}^{\prime} f$ converges to zero. We generalize the result of Impens and Gavrea [27], which was given for Bernstein type operators and for functions defined on a compact interval. Using another approach, we extend the result to larger class of positive linear operators and to a larger class of continuous functions, including bounded and unbounded functions. We also give a Voronovskaya type result for the differential formula just mentioned.

In Section 2, we present a class of positive linear operators which is defined using a ChebyshevGrüss expression. This class, which was introduced in [26], contains the Bernstein type operators [31], but is much larger, including also positive linear operators which do not preserve linear functions. Some examples are given in the final section of the paper as applications of the results obtained.

Received: 12.02.2020; Accepted: 23.03.2020; Published Online: 24.03.2020

*Corresponding author: Adrian Holhoş; Adrian.Holhos@math.utcluj.ro

DOI: $10.33205 / \mathrm{cma} .688661$ 


\section{Properties of THE OPERATORS DEFINED By A CHEBYSHEV-GRÜSS QUANTITY}

For the value of $L_{n} f$ in $x \in I$, we use the notations

$$
\left(L_{n} f\right)(x)=L_{n}(f)(x)=L_{n}(f, x)=L_{n}(f(t), x)
$$

interchangeably.

Consider a sequence of positive linear operators $\left(L_{n}\right)$ which preserve the constants and which is defined by the following relation involving a Chebyshev-Grüss type expression

$$
b_{n}\left[L_{n}(t f(t), x)-L_{n}(t, x) \cdot L_{n}(f(t), x)\right]=b(x)\left(L_{n} f\right)^{\prime}(x)
$$

for every $x \in I$, where $I \subset \mathbb{R}$ is an interval, for every $n \in \mathbb{N}$ and for every $f$ for which $L_{n} f$ and $\left(L_{n} f\right)^{\prime}$ exist, where $b(x)$ is a positive function which is differentiable on $I$ and $\left(b_{n}\right)$ is a sequence of positive real numbers such that $b_{n} \rightarrow \infty$. Concerning the domain of definition of the operators $L_{n}$, we will give explanations in the next section.

Remark 2.1. If the operators $L_{n}$ preserve the linear functions, then the condition (2.1) can be written

$$
\left(L_{n} f\right)^{\prime}(x)=\frac{b_{n}}{b(x)} \cdot L_{n}\left(\left(e_{1}-x\right) f, x\right),
$$

which is satisfied by the class of so called exponential operators (see [31] and [28]), in particular Bernstein polynomials, the operators of Szász-Mirakyan, Baskakov, Post-Widder and Gauss-Weierstrass. Condition (2.1) characterizes a more general class of operators, which do not necessarily preserve linear functions. Other examples will be given at the end of the paper. A relation equivalent with (2.1) is

$$
b_{n}\left[L_{n}((t-x) f(t), x)-L_{n}(t-x, x) \cdot L_{n}(f(t), x)\right]=b(x)\left(L_{n} f\right)^{\prime}(x),
$$

a relation obtained in [36] for a particular kind of operators.

Remark 2.2. If we consider a function $f=g(t, x)$ which depends on $x$ and $t$ and which has a partial derivative with respect to $x$ in every point $(t, x)$, then, condition (2.1) can be written

$$
\begin{aligned}
b_{n}\left[L_{n}((t-x) g(t, x), x)-L_{n}(t-x, x) \cdot L_{n}(g(t, x), x)\right] & \\
= & b(x)\left[\left(L_{n}(g(t, x), x)\right)^{\prime}-L_{n}\left(\frac{\partial g}{\partial x}(t, x), x\right)\right],
\end{aligned}
$$

where the operator $L_{n}$ acts on the variable $t$. In particular, for $f=(t-x)^{k}, k \geq 1$ we obtain

$$
b_{n} \cdot\left[\mu_{n, k+1}(x)-\mu_{n, 1}(x) \mu_{n, k}(x)\right]=b(x)\left[\mu_{n, k}^{\prime}(x)+k \mu_{n, k-1}(x)\right],
$$

where $\mu_{n, k}(x)=L_{n}\left((t-x)^{k}, x\right)$ are the central moments of order $k$ for the operator $L_{n}$. This recurrence expresses all the central moments in terms of only one function, namely $\mu_{n, 1}$, since the value of $\mu_{n, 0}$ is known: $\mu_{n, 0}(x)=1$.

Let us suppose that

$$
\lim _{n \rightarrow \infty} a_{n} \cdot \frac{d^{i}}{d x^{i}} \mu_{n, 1}(x)=a^{(i)}(x)
$$

is true for every $x \in I$, and $i=0,1,2, \ldots$, where $a(x)$ is an infinitely differentiable function on $I$ and $\left(a_{n}\right)$ is an increasing and unbounded sequence of positive real numbers. 
Lemma 2.1. If the sequence $\left(b_{n} / a_{n}\right)$ converges to the real number $c \geq 0$, then for every integer $\ell \geq 0$ we have

$$
\begin{aligned}
\lim _{n \rightarrow \infty} b_{n}^{\ell} \cdot \mu_{n, 2 \ell}(x) & =(b(x))^{\ell} \cdot(2 \ell-1) ! ! \\
\lim _{n \rightarrow \infty} b_{n}^{\ell+1} \cdot \mu_{n, 2 \ell+1}(x) & =(b(x))^{\ell}(2 \ell) ! ! \sum_{i=0}^{\ell} \frac{(2 i-1) ! !}{(2 i) ! !}\left[i b^{\prime}(x)+c \cdot a(x)\right]
\end{aligned}
$$

uniformly for $x$ in a compact interval contained in $I$. We have used the notations

$$
(2 \ell-1) ! !=1 \cdot 3 \ldots(2 \ell-1) \text { and }(2 \ell) ! !=2 \cdot 4 \cdots(2 \ell), \quad \ell \geq 1
$$

and for $\ell=0$ the value is 1 .

Proof. The proof will be omitted since it is similar to the one found in Lemma 2 [26].

\section{Quantitative Voronovskaya-TyPE Result fOR CHEBYSHEV-GRÜSS EXPRESSION}

In this section, we are concerned with the asymptotic behaviour of the Grüss-Chebyshev expression, which will be denoted

$$
T_{n}(f, g)(x)=L_{n}(f g)(x)-\left(L_{n} f\right)(x) \cdot\left(L_{n} g\right)(x) .
$$

We will prove that $b_{n} \cdot T_{n}(f, g)(x) \rightarrow b(x) f^{\prime}(x) g^{\prime}(x)$ and we will estimate the speed of this convergence. We show that such a result is valid for unbounded functions, too. In order to do this, let us introduce some notations.

Let $\theta:[0, \infty) \rightarrow \mathbb{R}$ be a uniformly continuous and monotonic function, let $I$ be an interval $I \subset \mathbb{R}$ and let $\alpha \geq 0$ be a real number. We denote by $C_{\theta, \alpha}$ the space of continuous functions $f \in C(I)$ with the property that exists $M>0$ such that $|f(x)| \leq M e^{\alpha \theta(|x|)}$, for every $x \in I$. Because of the symmetry and to simplify the notation, we consider in the following that $I \subset$ $[0, \infty)$. This space $C_{\theta, \alpha}$ can be endowed with the norm

$$
\|f\|_{\theta, \alpha}=\sup _{x \in I} e^{-\alpha \theta(x)}|f(x)| .
$$

Lemma 3.2. Consider a sequence of positive linear operators $L_{n}: C_{\theta, \alpha} \rightarrow C(I)$ such that

$$
\lim _{n \rightarrow \infty} L_{n}\left(e^{\alpha \theta(t)}, x\right)=e^{\alpha \theta(x)} .
$$

Then, there is a positive function $M_{\alpha}(x)$ not depending on $n$ such that

$$
L_{n}\left(\max \left(e^{\alpha \theta(t)}, e^{\alpha \theta(x)}\right), x\right) \leq M_{\alpha}(x), \quad n \geq n_{0} .
$$

Proof. For $x \in I$, there is $n_{0}$ such that $\left|L_{n}\left(e^{\alpha \theta(t)}, x\right)-e^{\alpha \theta(x)}\right| \leq 1$ for every $n \geq n_{0}$. We obtain

$$
L_{n}\left(\max \left(e^{\alpha \theta(t)}, e^{\alpha \theta(x)}\right), x\right) \leq L_{n}\left(e^{\alpha \theta(t)}+e^{\alpha \theta(x)}, x\right) \leq 1+2 e^{\alpha \theta(x)},
$$

for every $n \geq n_{0}$.

We will use the following weighted modulus

$$
\omega_{\theta, \alpha}(f, \delta)=\sup _{\substack{x, t \in I \\|t-x| \leq \delta}} \frac{|f(t)-f(x)|}{\max \left(e^{\alpha \theta(t)}, e^{\alpha \theta(x)}\right)},
$$

which is suitable for functions from the space $C_{\theta, \alpha}$ (see [25]). 
Theorem 3.1. Let $f, g \in C_{\theta, \alpha}$ be continuously differentiable functions such that $f^{\prime}(x) e^{-\alpha \theta(x)}$ and $g^{\prime}(x) e^{-\alpha \theta(x)}$ are uniformly continuous on $I$. Let $L_{n}: C_{\theta, \alpha} \rightarrow C^{1}(I)$ be a sequence of positive linear operators preserving constant functions and having the properties (2.1), (2.3) and (3.6). Then, for some $n_{0} \in \mathbb{N}$ and for every $n \geq n_{0}$ and $x \in I$, we have

$$
\begin{aligned}
&\left|b_{n}\left[L_{n}(f g)(x)-\left(L_{n} f\right)(x) \cdot\left(L_{n} g\right)(x)\right]-b(x) f^{\prime}(x) g^{\prime}(x)\right| \\
& \leq \frac{M(x)}{a_{n}}\left|b(x) f^{\prime}(x) g^{\prime}(x)\right|+M(x) \omega_{\theta, \alpha}\left(f^{\prime}, \frac{1}{\sqrt{b_{n}}}\right) \omega_{\theta, \alpha}\left(g^{\prime}, \frac{1}{\sqrt{b_{n}}}\right) \\
&+M(x)\left(\left|f^{\prime}(x)\right| \omega_{\theta, \alpha}\left(g^{\prime}, \frac{1}{\sqrt{b_{n}}}\right)+\left|g^{\prime}(x)\right| \omega_{\theta, \alpha}\left(f^{\prime}, \frac{1}{\sqrt{b_{n}}}\right)\right),
\end{aligned}
$$

where $M(x)>0$ does not depend on $n$ and $f$.

Proof. Using the Taylor formula of the first order with Lagrange remainder, we obtain

$$
\begin{array}{ll}
f(t)=f(x)+f^{\prime}(x) \cdot(t-x)+R_{f}, & R_{f}=\left(f^{\prime}\left(c_{1}\right)-f^{\prime}(x)\right) \cdot(t-x) \\
g(t)=g(x)+g^{\prime}(x) \cdot(t-x)+R_{g}, & R_{g}=\left(g^{\prime}\left(c_{2}\right)-g^{\prime}(x)\right) \cdot(t-x),
\end{array}
$$

with $c_{1}, c_{2}$ between $t$ and $x$. We multiply the relations and we apply the operators $L_{n}$. We get

$$
\begin{aligned}
L_{n}(f g)(x) & =f(x) g(x) \mu_{n, 0}(x)+\left[f(x) g^{\prime}(x)+g(x) f^{\prime}(x)\right] \mu_{n, 1}(x)+L_{n}\left(R_{f} R_{g}\right)(x) \\
& +f^{\prime}(x) g^{\prime}(x) \mu_{n, 2}(x)+f(x) L_{n}\left(R_{g}\right)(x)+g(x) L_{n}\left(R_{f}\right)(x) \\
& +f^{\prime}(x) L_{n}\left(\left(e_{1}-x e_{0}\right) R_{g}\right)(x)+g^{\prime}(x) L_{n}\left(\left(e_{1}-x e_{0}\right) R_{f}\right)(x) .
\end{aligned}
$$

We also have

$$
\begin{aligned}
& L_{n} f(x)=f(x) \mu_{n, 0}(x)+f^{\prime}(x) \mu_{n, 1}(x)+L_{n}\left(R_{f}\right)(x), \\
& L_{n} g(x)=g(x) \mu_{n, 0}(x)+g^{\prime}(x) \mu_{n, 1}(x)+L_{n}\left(R_{g}\right)(x) .
\end{aligned}
$$

We get

$$
\begin{aligned}
L_{n}(f g)(x)-\left(L_{n} f\right)(x) \cdot\left(L_{n} g\right)(x) & =f^{\prime}(x) g^{\prime}(x)\left[\mu_{n, 2}(x)-\mu_{n, 1}^{2}(x)\right] \\
& +f^{\prime}(x)\left[L_{n}\left((t-x) R_{g}\right)(x)-\mu_{n, 1}(x) L_{n}\left(R_{g}\right)(x)\right] \\
& +g^{\prime}(x)\left[L_{n}\left((t-x) R_{f}\right)(x)-\mu_{n, 1}(x) L_{n}\left(R_{f}\right)(x)\right] \\
& +L_{n}\left(R_{f} R_{g}\right)(x)-L_{n}\left(R_{f}\right)(x) \cdot L_{n}\left(R_{g}\right)(x) .
\end{aligned}
$$

Because $b_{n}\left[\mu_{n, 2}(x)-\mu_{n, 1}^{2}(x)\right]=b(x)\left[1+\mu_{n, 1}^{\prime}(x)\right]$, we have

$$
\left|b_{n}\left[\mu_{n, 2}(x)-\mu_{n, 1}^{2}(x)\right]-b(x)\right|=\left|b(x) \mu_{n, 1}^{\prime}(x)\right|=\frac{\left|b(x) a_{n} \mu_{n, 1}^{\prime}(x)\right|}{a_{n}} .
$$

We evaluate now the remainder from the Taylor formula using the modulus of continuity $\omega_{\theta, \alpha}$. From

$$
\begin{aligned}
\left|R_{f}\right| & =|t-x| \cdot\left|f^{\prime}\left(c_{1}\right)-f^{\prime}(x)\right| \\
& \leq \max \left(e^{\alpha \theta(t)}, e^{\alpha \theta(x)}\right)|t-x|\left(1+\frac{|t-x|}{\delta}\right) \omega_{\theta, \alpha}\left(f^{\prime}, \delta\right),
\end{aligned}
$$

we obtain

$$
\left|\left(L_{n} R_{f}\right)(x)\right| \leq\left(A_{n, 1}(x)+\sqrt{b_{n}} A_{n, 2}(x)\right) \omega_{\theta, \alpha}\left(f^{\prime}, \frac{1}{\sqrt{b_{n}}}\right)
$$

where

$$
A_{n, k}(x)=L_{n}\left(\max \left(e^{\alpha t}, e^{\alpha x}\right)|t-x|^{k}, x\right) .
$$


Because $L_{n}\left(e^{\alpha \theta(t)}, x\right)$ converges pointwise to $e^{\alpha \theta(x)}$ we have

$$
L_{n}\left(\max \left(e^{\alpha \theta(t)}, e^{\alpha \theta(x)}\right), x\right) \leq M_{\alpha}(x), \quad n \geq n_{0} .
$$

From Lemma 2.1, we have

$$
L_{n}\left(|t-x|^{2 k}, x\right)=\frac{1}{b_{n}^{k}} \cdot b_{n}^{k} \mu_{n, 2 k}(x) \leq \frac{C_{k}(x)}{b_{n}^{k}}, \quad n \geq n_{0} .
$$

Using the Cauchy-Schwarz inequality for positive linear operators we obtain for $k=1,2$

$$
A_{n, k}(x) \leq \sqrt{L_{n}\left(\max \left(e^{2 \alpha t}, e^{2 \alpha x}\right), x\right)} \cdot \sqrt{L_{n}\left(|t-x|^{2 k}, x\right)} \leq \frac{\sqrt{M_{2 \alpha}(x) C_{k}(x)}}{\sqrt{b_{n}^{k}}} .
$$

In conclusion,

Similarly,

$$
\left|\left(L_{n} R_{f}\right)(x)\right| \leq \frac{M_{0,2}(x)}{\sqrt{b_{n}}} \cdot \omega_{\theta, \alpha}\left(f^{\prime}, \frac{1}{\sqrt{b_{n}}}\right)
$$

$$
\left|L_{n}\left(\left(e_{1}-x e_{0}\right) R_{f}, x\right)\right| \leq L_{n}\left(|t-x|\left|R_{f}\right|, x\right) \leq \frac{M_{0,3}(x)}{b_{n}} \cdot \omega_{\theta, \alpha}\left(f^{\prime}, \frac{1}{\sqrt{b_{n}}}\right) .
$$

So, using $\left|\mu_{n, 1}(x)\right| \leq \sqrt{\mu_{n, 2}(x)} \leq \sqrt{C_{2}(x)} / \sqrt{b_{n}}$, we obtain

$$
\begin{aligned}
\mid L_{n}\left(\left(e_{1}-\right.\right. & \left.\left.x e_{0}\right) R_{f}\right)(x)-\mu_{n, 1}(x) L_{n}\left(R_{f}\right)(x) \mid \\
& \leq\left|L_{n}\left(\left(e_{1}-x e_{0}\right) R_{f}, x\right)\right|+\left|\mu_{n, 1}(x)\right| \cdot\left|\left(L_{n} R_{f}\right)(x)\right| \\
& \leq \frac{M_{0,4}(x)}{b_{n}} \cdot \omega_{\theta, \alpha}\left(f^{\prime}, \frac{1}{\sqrt{b_{n}}}\right) .
\end{aligned}
$$

Let us notice that if we replace $f$ with $g$ in the previous inequalities they hold true, too.

To evaluate the term $L_{n}\left(R_{f} R_{g}\right)(x)-L_{n}\left(R_{f}\right)(x) \cdot L_{n}\left(R_{g}\right)(x)$, let us observe that

$$
\begin{aligned}
& \left|L_{n}\left(R_{f} R_{g}\right)(x)\right| \leq L_{n}\left(\left|R_{f}\right|\left|R_{g}\right|, x\right) \\
& \leq L_{n}\left(e^{2 \alpha \max (\theta(t), \theta(x))}|t-x|^{2}\left(1+\frac{|t-x|}{\delta_{n}}\right)^{2}, x\right) \cdot \omega_{\theta, \alpha}\left(f^{\prime}, \delta_{n}\right) \cdot \omega_{\theta, \alpha}\left(g^{\prime}, \delta_{n}\right) \\
& \leq 2\left[A_{n, 2}(x)+b_{n} A_{n, 4}(x)\right] \cdot \omega_{\theta, \alpha}\left(f^{\prime}, \frac{1}{\sqrt{b_{n}}}\right) \omega_{\theta, \alpha}\left(g^{\prime}, \frac{1}{\sqrt{b_{n}}}\right) \\
& \leq \frac{M_{0,5}(x)}{b_{n}} \cdot \omega_{\theta, \alpha}\left(f^{\prime}, \frac{1}{\sqrt{b_{n}}}\right) \omega_{\theta, \alpha}\left(g^{\prime}, \frac{1}{\sqrt{b_{n}}}\right) .
\end{aligned}
$$

We have used the inequality $(1+u)^{2} \leq 2\left(1+u^{2}\right)$. We obtain

$$
\begin{aligned}
& \left|L_{n}\left(R_{f} R_{g}\right)(x)-L_{n}\left(R_{f}\right)(x) \cdot L_{n}\left(R_{g}\right)(x)\right| \\
& \leq\left|L_{n}\left(R_{f} R_{g}\right)(x)\right|+\left|L_{n}\left(R_{f}\right)(x)\right| \cdot\left|L_{n}\left(R_{g}\right)(x)\right| \\
& \quad \leq \frac{M_{0,5}(x)+M_{0,2}^{2}(x)}{b_{n}} \cdot \omega_{\theta, \alpha}\left(f^{\prime}, \frac{1}{\sqrt{b_{n}}}\right) \omega_{\theta, \alpha}\left(g^{\prime}, \frac{1}{\sqrt{b_{n}}}\right) .
\end{aligned}
$$

Choosing an appropriate expression $M(x)>0$ not depending on $n$ and $f$ the proof is complete.

Remark 3.3. Because $1 / a_{n}$ and $1 / \sqrt{b_{n}}$ converge to zero when $n$ tends to infinity and $f^{\prime}(x) e^{-\alpha \theta(x)}$ and $g^{\prime}(x) e^{-\alpha \theta(x)}$ are uniformly continuous on $I$, we have

$$
\lim _{n \rightarrow \infty} b_{n}\left[L_{n}(f g)(x)-\left(L_{n} f\right)(x) \cdot\left(L_{n} g\right)(x)\right]=b(x) f^{\prime}(x) g^{\prime}(x) .
$$


Similar results were obtained in $[18,6,16,35,9,30]$ for functions for which the second derivative exists. $B u t$, there is no need to suppose the existence of the second derivative of $f$ and $g$.

Remark 3.4. Theorem 3.1 is true even for operators $L_{n}$ for which (2.1) cannot be proved. Indeed, it is only necessary that the following limits exist for a fixed $x$

$$
\lim _{n \rightarrow \infty} a_{n}\left(b_{n}\left[\mu_{n, 2}(x)-\mu_{n, 1}^{2}(x)\right]-b(x)\right) \text { and } \lim _{n \rightarrow \infty} b_{n}^{\ell} \cdot \mu_{n, 2 \ell}(x), \ell=1,2,3,4,
$$

where $b(x)$ is the limit of $b_{n} \cdot \mu_{n, 2}(x)$.

For example, let us consider the Jain operators [29]

$$
P_{n}^{\beta_{n}}(f, x)=\sum_{k=0}^{\infty} \frac{n x\left(n x+k \beta_{n}\right)^{k-1}}{k !} e^{-n x-k \beta_{n}} \cdot f\left(\frac{k}{n}\right),
$$

where $\left(\beta_{n}\right)$ is a sequence of positive real numbers from $[0,1)$ converging to zero. It is known [17] that

$$
P_{n}^{\beta_{n}}(t-x, x)=\frac{x}{1-\beta_{n}}-x=\frac{x \beta_{n}}{1-\beta_{n}},
$$

so we choose $a_{n}=1 / \beta_{n}$ and condition (2.3) is satisfied with $a(x)=x$. We also have

$$
P_{n}^{\beta_{n}}\left((t-x)^{2}, x\right)=\frac{x^{2} \beta_{n}^{2}}{\left(1-\beta_{n}\right)^{2}}+\frac{x}{n\left(1-\beta_{n}\right)^{3}} .
$$

Choosing $b_{n}=n$ and supposing that $b_{n} / a_{n}=n \beta_{n}$ is convergent to the real number $c \geq 0$, we obtain

$$
b(x)=\lim _{n \rightarrow \infty} n P_{n}^{\beta_{n}}\left((t-x)^{2}, x\right)=x .
$$

After some computations, we obtain

$$
\lim _{n \rightarrow \infty} a_{n}\left(b_{n}\left[\mu_{n, 2}(x)-\mu_{n, 1}^{2}(x)\right]-b(x)\right)=x .
$$

The central moment of order 4 is (see [17])

$$
\begin{aligned}
P_{n}^{\beta_{n}}\left((t-x)^{4}, x\right)= & \frac{x^{4} \beta_{n}^{4}}{\left(1-\beta_{n}\right)^{4}}+\frac{6 x^{3} \beta_{n}^{2}}{n\left(1-\beta_{n}\right)^{5}} \\
& +\frac{x^{2}\left(-24 \beta_{n}^{5}+12 \beta_{n}+48 \beta_{n}^{3}-28 \beta_{n}^{2}+4 \beta_{n}+3\right)}{n^{2}\left(1-\beta_{n}\right)^{6}} \\
& +\frac{x\left(105 \beta_{n}^{5}-14 \beta_{n}^{4}-2 \beta_{n}^{3}+12 \beta_{n}^{2}+8 \beta_{n}+1\right)}{n^{3}\left(1-\beta_{n}\right)^{7}} .
\end{aligned}
$$

We obtain $n^{2} P_{n}^{\beta_{n}}\left((t-x)^{4}, x\right) \rightarrow 3 x^{2}$. For the central moments of order 6 and 8 , we consider the significant terms from the formulas given in [23] and obtain

$$
\lim _{n \rightarrow \infty} n^{3} P_{n}^{\beta_{n}}\left((t-x)^{6}, x\right)=15 x^{3} \text { and } \lim _{n \rightarrow \infty} n^{4} P_{n}^{\beta_{n}}\left((t-x)^{8}, x\right)=105 x^{4} .
$$

The result of Theorem 3.1 is valid for $P_{n}^{\beta_{n}}$ in polynomial weighted space $C_{\theta, \alpha}$ with $\theta(x)=\ln x, x \in$ $I=(0, \infty)$ (see [2]).

\section{VORONOVSKAYA-TYPE RESULT FOR A DIFFERENTIATION FORMULA FOR POSITIVE LINEAR OPERATORS}

In [27], it is proved that the expression $L_{n}^{\prime}(f g)-f L_{n}^{\prime} g-g L_{n}^{\prime} f$ converges to zero for exponential type operators under suitable conditions for the functions $f$ and $g$. The result was proved for those operators $L_{n}: C(I) \rightarrow C(J)$, where $I, J$ are compact intervals. We extend the result to noncompact intervals and to unbounded functions. 
Theorem 4.2. Let $f, g \in C_{\theta, \alpha}$ such that

$$
\omega_{\theta, \alpha}(f, \delta) \cdot \omega_{\theta, \alpha}(g, \delta)=o(\delta) \quad(\delta \rightarrow 0+) .
$$

Let $L_{n}: C_{\theta, \alpha} \rightarrow C^{1}(I)$ be a sequence of positive linear operators preserving constant functions and having the properties (2.1), (2.3) and (3.6). Then, for every $x \in I$

$$
L_{n}^{\prime}(f g)(x)-f(x)\left(L_{n} g\right)^{\prime}(x)-g(x)\left(L_{n} f\right)^{\prime}(x) \rightarrow 0 .
$$

Proof. Let us denote

$$
\Delta_{n}(x)=L_{n}^{\prime}(f g)(x)-f(x)\left(L_{n} g\right)^{\prime}(x)-g(x)\left(L_{n} f\right)^{\prime}(x) .
$$

Using (2.1), we obtain the following relation

$$
\begin{aligned}
\Delta_{n}(x)= & \frac{b_{n}}{b(x)} \cdot L_{n}((t-x)(f(t)-f(x))(g(t)-g(x)), x) \\
& -\frac{b_{n}}{b(x)} \cdot L_{n}(t-x, x) \cdot L_{n}((f(t)-f(x))(g(t)-g(x)), x) .
\end{aligned}
$$

As in the proof of Theorem 3.1, because

$$
\begin{aligned}
& |f(t)-f(x)| \leq \max \left(e^{\alpha \theta(t)}, e^{\alpha \theta(x)}\right)\left(1+\frac{|t-x|}{\delta}\right) \omega_{\theta, \alpha}(f, \delta) \\
& |g(t)-g(x)| \leq \max \left(e^{\alpha \theta(t)}, e^{\alpha \theta(x)}\right)\left(1+\frac{|t-x|}{\delta}\right) \omega_{\theta, \alpha}(g, \delta),
\end{aligned}
$$

we have

$$
\begin{aligned}
\mid L_{n}((t-x) & (f(t)-f(x))(g(t)-g(x)), x) \mid \\
\leq & 2\left(A_{n, 1}(x)+b_{n} A_{n, 3}(x)\right) \omega_{\theta, \alpha}\left(f, \frac{1}{\sqrt{b_{n}}}\right) \omega_{\theta, \alpha}\left(g, \frac{1}{\sqrt{b_{n}}}\right) \\
\leq & \frac{M_{1,1}(x)}{\sqrt{b_{n}}} \cdot \omega_{\theta, \alpha}\left(f, \frac{1}{\sqrt{b_{n}}}\right) \omega_{\theta, \alpha}\left(g, \frac{1}{\sqrt{b_{n}}}\right)
\end{aligned}
$$

and

$$
\begin{aligned}
\mid L_{n}((f(t) & -f(x))(g(t)-g(x)), x) \mid \\
& \leq 2\left(A_{n, 0}(x)+b_{n} A_{n, 2}(x)\right) \omega_{\theta, \alpha}\left(f, \frac{1}{\sqrt{b_{n}}}\right) \omega_{\theta, \alpha}\left(g, \frac{1}{\sqrt{b_{n}}}\right) \\
& \leq M_{1,2}(x) \cdot \omega_{\theta, \alpha}\left(f, \frac{1}{\sqrt{b_{n}}}\right) \omega_{\theta, \alpha}\left(g, \frac{1}{\sqrt{b_{n}}}\right) .
\end{aligned}
$$

Because $L_{n}(t-x, x) \leq \sqrt{\mu_{n, 2}(x)} \leq \frac{\sqrt{C_{2}(x)}}{\sqrt{b_{n}}}$, we finally obtain

$$
\left|\Delta_{n}(x)\right| \leq M(x) \sqrt{b_{n}} \cdot \omega_{\theta, \alpha}\left(f, \frac{1}{\sqrt{b_{n}}}\right) \omega_{\theta, \alpha}\left(g, \frac{1}{\sqrt{b_{n}}}\right), \quad n \geq n_{0},
$$

for some $M(x)$ not depending on $n$ and $f$. The condition (4.9) proves that $\Delta_{n}$ converges to zero for every $x \in I$.

Remark 4.5. We have the following evaluation of the modulus $\omega_{\theta, \alpha}$ (see relation (1) from [25])

$$
\omega_{\theta, \alpha}(f, \delta) \leq\left(1-e^{-\alpha \omega(\theta, \delta)}\right)\|f\|_{\theta, \alpha}+\omega(f / w, \delta) \leq \alpha \omega(\theta, \delta)\|f\|_{\theta, \alpha}+\omega(f / w, \delta),
$$

where $w(x)=e^{\alpha \theta(x)}$ and $\omega$ is the usual modulus of continuity (the modulus $\omega_{\theta, \alpha}$ for $\alpha=0$ ). 
If $\theta \in \operatorname{Lip}_{a}(I), f / w \in \operatorname{Lip}_{b}(I)$ and $g / w \in \operatorname{Lip}_{c}(I)$ then, (4.9) is true if and only if

$$
a+a>1, \quad a+b>1, \quad a+c>1 \text { and } b+c>1 .
$$

Indeed, a function $h$ belongs to $\operatorname{Lip}_{\alpha}(I)$ if and only if there is a constant $C_{h}>0$ such that $\omega(f, \delta) \leq$ $C_{h} \delta^{\alpha}$. So,

$$
\omega_{\theta, \alpha}(f, \delta) \cdot \omega_{\theta, \alpha}(g, \delta) \leq\left(C_{1} \delta^{a}+C_{2} \delta^{b}\right)\left(C_{1} \delta^{a}+C_{3} \delta^{c}\right)=o(\delta) \quad(\delta \rightarrow 0+) .
$$

Remark 4.6. Theorem 4.2 remains true even if $L_{n}$ does not satisfy a condition like (2.1). We only need that the sequence of functions $b_{n}^{\ell} \cdot \mu_{n, 2 \ell}(x)$ converges pointwise for $\ell=1,2$ and 3 .

Theorem 4.3. Let $f, g \in C_{\theta, \alpha}$ be two twice differentiable functions such that $f^{\prime \prime}(x) e^{-\alpha \theta(x)}$ and $g^{\prime \prime}(x) e^{-\alpha \theta(x)}$ are uniformly continuous on $I$. We suppose that $\left(b_{n} / a_{n}\right)$ is convergent to $c \geq 0$. Let $L_{n}: C_{\theta, \alpha} \rightarrow C^{1}(I)$ be a sequence of positive linear operators preserving constant functions and having the properties (2.1), (2.3) and (3.6). Then, for every $x \in I$

$$
\begin{aligned}
\lim _{n \rightarrow \infty} b_{n} & {\left[L_{n}^{\prime}(f g)(x)-f(x)\left(L_{n} g\right)^{\prime}(x)-g(x)\left(L_{n} f\right)^{\prime}(x)\right] } \\
= & {\left[b^{\prime}(x)+2 c a(x)\right] f^{\prime}(x) g^{\prime}(x)+\frac{3 b(x)}{2}\left[f^{\prime}(x) g^{\prime \prime}(x)+f^{\prime \prime}(x) g^{\prime}(x)\right] . }
\end{aligned}
$$

Proof. We use Taylor's formula

$$
h(t)=h(x)+h^{\prime}(x) \cdot(t-x)+\frac{h^{\prime \prime}(x)}{2} \cdot(t-x)^{2}+R_{h},
$$

for the functions $f$ and $g$, where $R_{h}=\left(h^{\prime \prime}(c)-h^{\prime \prime}(x)\right) \cdot(t-x)^{2} / 2$, with some $c$ between $t$ and $x$. We replace these formulas in the expression of $\Delta_{n}$ (see relation (4.10)) and after some computations, we obtain

$$
\begin{aligned}
b_{n} \Delta_{n}(x) & =f^{\prime}(x) g^{\prime}(x) \frac{b_{n}^{2}}{b(x)}\left[\mu_{n, 3}(x)-\mu_{n, 1}(x) \mu_{n, 2}(x)\right] \\
& +\left[f^{\prime}(x) g^{\prime \prime}(x)+f^{\prime \prime}(x) g^{\prime}(x)\right] \cdot \frac{b_{n}^{2}}{2 b(x)}\left[\mu_{n, 4}(x)-\mu_{n, 1}(x) \mu_{n, 3}(x)\right]+\frac{b_{n}^{2} R}{b(x)},
\end{aligned}
$$

where

$$
\begin{aligned}
R & =\frac{1}{4} f^{\prime \prime}(x) g^{\prime \prime}(x)\left[\mu_{n, 5}(x)-\mu_{n, 1}(x) \mu_{n, 5}(x)\right] \\
& +f^{\prime}(x) \cdot E_{n, 1}(g)+\frac{1}{2} f^{\prime \prime}(x) \cdot E_{n, 2}(g)+g^{\prime}(x) \cdot E_{n, 1}(f)+\frac{1}{2} g^{\prime \prime}(x) \cdot E_{n, 2}(f) \\
& +L_{n}\left(R_{f} \cdot R_{g} \cdot(t-x), x\right)-\mu_{n, 1}(x) \cdot L_{n}\left(R_{f} \cdot R_{g}, x\right)
\end{aligned}
$$

and

$$
E_{n, k}(f)=L_{n}\left(R_{f} \cdot(t-x)^{k+1}, x\right)-\mu_{n, 1}(x) \cdot L_{n}\left(R_{f} \cdot(t-x)^{k}, x\right)
$$

We have

$$
\frac{b_{n}^{2}}{b(x)}\left[\mu_{n, 3}(x)-\mu_{n, 1}(x) \mu_{n, 2}(x)\right]=b_{n} \mu_{n, 2}^{\prime}(x)+2 b_{n} \mu_{n, 1}(x) \rightarrow b^{\prime}(x)+2 c a(x)
$$

and

$$
\frac{b_{n}^{2}}{2 b(x)}\left[\mu_{n, 4}(x)-\mu_{n, 1}(x) \mu_{n, 3}(x)\right]=\frac{b_{n}}{2} \mu_{n, 3}^{\prime}(x)+\frac{3 b_{n}}{2} \mu_{n, 2}(x) \rightarrow 0+\frac{3 b(x)}{2} .
$$

We also have $b_{n}^{2} R \rightarrow 0$, but since the computations are similar to those in the proof of Theorem 3.1, we omit the details. 
Remark 4.7. Let $f, g \in C_{\theta, \alpha}$ be two twice differentiable functions such that $f^{\prime \prime}(x) e^{-\alpha \theta(x)}$ and $g^{\prime \prime}(x) e^{-\alpha \theta(x)}$ are uniformly continuous on $I$. It can be proved in a similar way that

$$
\begin{gathered}
\lim _{n \rightarrow \infty} b_{n}\left[L_{n}^{\prime}(f g)(x)-\left(L_{n} f\right)(x)\left(L_{n} g\right)^{\prime}(x)-\left(L_{n} g\right)(x)\left(L_{n} f\right)^{\prime}(x)\right] \\
=b^{\prime}(x) f^{\prime}(x) g^{\prime}(x)+b(x)\left[f^{\prime}(x) g^{\prime \prime}(x)+f^{\prime \prime}(x) g^{\prime}(x)\right] .
\end{gathered}
$$

This is just relation (3.8), where both terms have been differentiated.

\section{Applications}

We give a couple of examples of applications.

Example 5.1. Consider the following Baskakov operators of Stancu type

$$
\left(L_{n}^{[\alpha, \beta, c]} f\right)(x)=\sum_{k=0}^{\infty} p_{n, k}^{[c]}(x) \cdot f\left(\frac{k+\alpha}{n+\beta}\right), \quad n \geq 1
$$

where $\alpha$ and $\beta$ are real numbers such that $0 \leq \alpha \leq \beta$ and

$$
\begin{aligned}
& p_{n, k}^{[c]}(x)=(-1)^{k}\left(\begin{array}{c}
-n / c \\
k
\end{array}\right)(c x)^{k}(1+c x)^{-\frac{n}{c}-k}, \quad c \neq 0 \\
& p_{n, k}^{[0]}(x)=\lim _{c \rightarrow 0} p_{n, k}^{[c]}(x)=\frac{(n x)^{k}}{k !} e^{-n x},
\end{aligned}
$$

where $x \in[0, \infty)$ for $c \geq 0$ and $x \in[0,-1 / c]$ for $c<0$.

These operators are a particular example of the more general operators considered in [11]. For $\alpha=$ $\beta=0$, some properties of the operators were given in $[1,14]$ (see also $[5,32]$ and the references therein).

These operators preserve the constants and

$$
L_{n}^{[\alpha, \beta, c]}(t, x)=\sum_{k=0}^{\infty} p_{n, k}^{[c]}(x) \cdot \frac{k+\alpha}{n+\beta}=\frac{n x+\alpha}{n+\beta} .
$$

We deduce that

$$
(n+\beta) \cdot\left(L_{n}^{[\alpha, \beta, c]}(t, x)-x\right)=\alpha-\beta x,
$$

which proves (2.3) for $a_{n}=n+\beta$ and $a(x)=\alpha-\beta x$.

We also have

$$
\frac{\mathrm{d}}{\mathrm{d} x} p_{n, k}^{[c]}(x)=p_{n, k}^{[c]}(x) \cdot \frac{k-n x}{x(1+c x)}=\frac{n+\beta}{x(1+c x)} p_{n, k}^{[c]}(x) \cdot\left(\frac{k+\alpha}{n+\beta}-L_{n}^{[\alpha, \beta, c]}(t, x)\right) .
$$

Multiplying this equality with $f((k+\alpha) /(n+\beta))$ and summing up for $k$ from 0 to infinity, we obtain

$$
\frac{\mathrm{d}}{\mathrm{d} x}\left(L_{n}^{[\alpha, \beta, c]} f\right)(x)=\frac{n+\beta}{x(1+c x)}\left[L_{n}^{[\alpha, \beta, c]}(t f(t), x)-L_{n}^{[\alpha, \beta, c]}(t, x) \cdot L_{n}^{[\alpha, \beta, c]}(f, x)\right],
$$

which is (2.1) for $b_{n}=n+\beta$ and $b(x)=x(1+c x)$.

The results of Theorems 1,2 and 3 are valid for functions in the exponential space $C_{\theta, \alpha}$ for $\theta(x)=x$, because

$$
L_{n}^{[\alpha, \beta, c]}\left(e^{\alpha t}, x\right)=\left(1+c x-c x e^{\frac{\alpha}{n}}\right)^{-\frac{n}{c}} \rightarrow e^{\alpha x} \quad(n \rightarrow \infty) .
$$

Example 5.2. Consider the Balász operators

$$
R_{n}(f, x)=\frac{1}{\left(1+\alpha_{n} x\right)^{n}} \sum_{k=0}^{n}\left(\begin{array}{l}
n \\
k
\end{array}\right)\left(\alpha_{n} x\right)^{k} \cdot f\left(\frac{k}{\beta_{n}}\right), n \geq 1
$$

introduced in [12] and studied in [13, 34, 4, 8, 3] for some particular cases of the sequences $\left(\alpha_{n}\right)$ and $\left(\beta_{n}\right)$ of positive real numbers. 
The operators $R_{n}$ preserve the constants and

$$
R_{n}(t, x)=\frac{n \alpha_{n} x}{\beta_{n}\left(1+\alpha_{n} x\right)} \text { and } R_{n}\left(t^{2}, x\right)=\frac{n \alpha_{n} x+n^{2} \alpha_{n}^{2} x^{2}}{\beta_{n}^{2}\left(1+\alpha_{n} x\right)^{2}} .
$$

We must have $R_{n}(t, x) \rightarrow x$ and $R_{n}\left(t^{2}, x\right) \rightarrow x^{2}$, so we choose $\left(\alpha_{n}\right)$ and $\left(\beta_{n}\right)$ such that $\alpha_{n} \rightarrow 0$ and the sequence $\left(\beta_{n}\right)$ such that $\beta_{n} \rightarrow \infty$ and $n \alpha_{n} / \beta_{n} \rightarrow 1$. The central moment of order 1 is

$$
R_{n}(t-x, x)=\frac{\left(n \alpha_{n}-\beta_{n}\right) x-\alpha_{n} \beta_{n} x^{2}}{\beta_{n}\left(1+\alpha_{n} x\right)} .
$$

We further impose that $n \alpha_{n}-\beta_{n} \rightarrow 0$ and $\alpha_{n} \beta_{n} \rightarrow c, c \geq 0$. With these conditions, we can choose $a_{n}=\beta_{n}$ and obtain $\beta_{n} R_{n}(t-x, x)=-c x^{2}$.

Let us prove that $R_{n}$ satisfy (2.1). Because

$$
\left(\frac{\left(\alpha_{n} x\right)^{k}}{\left(1+\alpha_{n} x\right)^{n}}\right)^{\prime}=\frac{\left(\alpha_{n} x\right)^{k}}{\left(1+\alpha_{n} x\right)^{n}} \cdot\left(\frac{k}{x}-\frac{n \alpha_{n}}{1+\alpha_{n} x}\right),
$$

we obtain

$$
\begin{aligned}
\left(R_{n}(f, x)\right)^{\prime} & =\frac{\beta_{n}}{x} \sum_{k=0}^{n}\left(\begin{array}{l}
n \\
k
\end{array}\right) \frac{\left(\alpha_{n} x\right)^{k}}{\left(1+\alpha_{n} x\right)^{n}} \cdot\left(\frac{k}{\beta_{n}}-\frac{n \alpha_{n} x}{\beta_{n}\left(1+\alpha_{n} x\right)}\right) \cdot f\left(\frac{k}{\beta_{n}}\right) \\
& =\frac{\beta_{n}}{x} R_{n}\left(\left(t-R_{n}(t, x)\right) f(t), x\right),
\end{aligned}
$$

which proves (2.1) with $b_{n}=\beta_{n}$ and $b(x)=x$.

We take $I=(0, \infty)$ and $\theta(x)=x$. The results of Theorem 1,2 and 3 are valid for the operators $R_{n}$ in the exponential weighted space $C_{\theta, \alpha}$, because for a fixed $x \geq 0$

$$
R_{n}\left(e^{\alpha t}, x\right)=\left(\frac{1+\alpha_{n} x e^{\frac{\alpha}{\beta_{n}}}}{1+\alpha_{n} x}\right)^{n} \rightarrow e^{\alpha x}
$$

\section{REFERENCES}

[1] U. Abel: An identity for a general class of approximation operators. J. Approx. Theory 142 (2006), 20-35.

[2] U. Abel, O. Agratini: Asymptotic behaviour of Jain operators. Numer. Algor. 71 (2016), 553-565.

[3] U. Abel, O. Agratini: On the variation detracting property of operators of Balázs and Szabados. Acta Math. Hungar. 150 (2016), 383-395.

[4] U. Abel, B. della Vecchia: Asymptotic approximation by the operators of K. Balázs and Szabados. Acta Sci. Math. (Szeged) 66 (1-2) (2000), 137-145.

[5] U. Abel, W. Gawronski and T. Neuschel: Complete monotonicity and zeros of sums of squared Baskakov functions. Appl. Math. Comput. 258 (2015), 130-137.

[6] T. Acar: Quantitative q-Voronovskaya and q-Grüss-Voronovskaya-type results for q-Szász operators. Georgian Math. J. 23 (2016), 459-468.

[7] A. M. Acu, H. Gonska and I. Raşa: Grüss-type and Ostrowski-type in approximation theory. Ukr. Math. J. 63 (2011), 843-864.

[8] O. Agratini: On approximation properties of Balázs-Szabados operators and their Kantorovich extension. Korean J. Comput. \& Appl. Math. 9 (2002), 361-372.

[9] O. Agratini: Properties of discrete non-multiplicative operators. Anal. Math. Phys. 9 (2019), 131-141.

[10] D. Andrica, C. Badea: Grüss inequality for positive linear functionals. Period. Math. Hungar. 19 (1988), 155-167.

[11] Ç. Atakut: On the approximation of functions together with derivatives by certain linear positive operators. Commun. Fac. Sci. Univ. Ank. Sér. A1 Math. Stat. 46 (1997), 57-65.

[12] K. Balázs: Approximation by Bernstein type rational functions. Acta Math. Acad. Sci. Hungar. 26 (1975), 123-134.

[13] C. Balázs, J. Szabados: Approximation by Bernstein type rational functions. II. Acta Math. Acad. Sci. Hungar. 40 (1982), 331-337.

[14] E. Berdysheva: Studying Baskakov-Durrmeyer operators and quasi-interpolants via special functions. J. Approx. Theory 149 (2007), 131-150. 
[15] P. L. Chebyshev: Sur les expressions approximatives des integrales définies par les autres prises entre les même limites. Proc. Math. Soc. Kharkov 2 (1882), 93-98.

[16] E. Deniz: Quantitative estimates for Jain-Kantorovich operators. Commun. Fac. Sci. Univ. Ank. Sér. A1 Math. Stat. 65 (2016), 121-132.

[17] A. Farcaş: An asymptotic formula for Jain's operators. Stud. Univ. Babeş-Bolyai Math. 57 (2012), 511-517.

[18] S. G. Gal, H. Gonska: Grüss and Grüss-Voronovskaya-type estimates for some Bernstein-type polynomials of real and complex variables. Jaen J. Approx. 7 (2015), 97-122.

[19] B. Gavrea, I. Gavrea: Ostrowski type inequalities from a linear functional point of view. J. Inequal. Pure Appl. Math. 1 (2000), Article 11.

[20] H. Gonska, I. Raşa and M. D. Rusu: Čebyšev-Grüss inequalities revisited. Math. Slov. 63 (2013), 1007-1024.

[21] H. Gonska, I. Raşa and M. D. Rusu: Chebyshev-Grüss-type inequalities via discrete oscillations. Bul. Acad. Ştiinţe Repub. Mold. Mat. 1 (74) (2014), 63-89.

[22] H. Gonska, G. Tachev: Grüss type inequality for positive linear operators with second order moduli. Mat. Vesn. 63 (2011), $247-252$.

[23] G. C. Greubel: A note on Jain basis functions. arXiv:1612.09385 [math.CA], (2016).

[24] G. Grüss: Über das Maximum des Absoluten Betrages von $\frac{1}{b-a} \int_{a}^{b} f(x) g(x) d x-\frac{1}{(b-a)^{2}} \int_{a}^{b} f(x) d x \int_{a}^{b} g(x) d x$. Math. Z. 39 (1935), 215-226.

[25] A. Holhoş: Quantitative Estimates of Voronovskaya Type in Weighted Spaces. Results Math. 73 (2018), 53.

[26] A. Holhoş: A Voronovskaya-Type Theorem for the First Derivatives of Positive Linear Operators. Results Math. 74 (2019), 76, https://doi.org/10.1007/s00025-019-0992-0.

[27] C. Impens, I. Gavrea: A Leibniz differentiation formula for positive operators. J. Math. Anal. Appl. 271 (2002), 175-181.

[28] M. E. H. Ismail, C. P. May: On a Family of Approximation Operators. J. Math. Anal. Appl. 63 (1978), 446-462.

[29] G. C. Jain: Approximation of functions by a new class of linear operators. J. Aust. Math. Soc. 13 (1972), 271-276.

[30] A. Kajla, S. Deshwal and P. N. Agrawal: Quantitative Voronovskaya and Grüss-Voronovskaya type theorems for Jain-Durrmeyer operators of blending type. Anal. Math. Phys. 9 (2019), 1241-1263.

[31] C. P. May: Saturation and inverse theorems for combinations of a class of exponential-type operators. Canad. J. Math 28 (1976), 1224-1250.

[32] I. Raşa: Entropies and Heun functions associated with positive linear operators. Appl. Math. Comput. 268 (2015), $422-$ 431.

[33] M. D. Rusu: On Grüss-type inequalities for positive linear operators. Stud. Univ. Babeş-Bolyai Math. 56 (2011), 551565.

[34] V. Totik: Saturation for Bernstein type rational functions. Acta Math. Hungar. 43 (1984), 219-250.

[35] G. Ulusoy, T. Acar: q-Voronovskaya type theorems for q-Baskakov operators. Math. Methods Appl. Sci. 39 (2016), 3391-3401.

[36] A. Wafi, S. Khatoon: Convergence and Voronovskaja-type theorems for derivatives of generalized Baskakov operators. Cent. Eur. J. Math. 6 (2008), 325-334.

\author{
TECHNICAL UNIVERSITY OF CLUJ-NAPOCA \\ DEPARTMENT OF MATHEMATICS \\ STR. MEMORANDUMUlui 28, RO-400114, CLUJ-NAPOCA, Romania \\ ORCID: 0000-0002-9695-9157 \\ E-mail address: Adrian. Holhos@math.utcluj.ro
}

